\title{
HARMONIC LASING IN X-RAY FELS: THEORY AND EXPERIMENT
}

\author{
E. A. Schneidmiller*, B. Faatz, M. Kuhlmann, J. Rönsch-Schulenburg, \\ S. Schreiber, M. Tischer, M .V. Yurkov \\ Deutsches Elektronen-Synchrotron (DESY), Notkestrasse 85, D-22607 Hamburg, Germany
}

\section{Abstract}

Harmonic lasing is a perspective mode of operation of $\mathrm{X}$ ray FEL user facilities that allows to provide brilliant beams of higher energy photons for user experiments. Another useful application of harmonic lasing is so called Harmonic Lasing Self-Seeded Free Electron Laser (HLSS FEL) that allows to improve spectral brightness of these facilities. In the past, harmonic lasing has been demonstrated in the FEL oscillators in infrared and visible wavelength ranges, but not in high-gain FELs and not at short wavelengths. In this paper we report on the first evidence of the harmonic lasing and the first operation of the HLSS FEL at the soft X-ray FEL user facility FLASH in the wavelength range between $4.5 \mathrm{~nm}$ and $15 \mathrm{~nm}$.

\section{INTRODUCTION}

Successful operation of X-ray free electron lasers (FELs) [1-3], based on self-amplified spontaneous emission (SASE) principle [4], down to an Ångström regime opens up new horizons for photon science. Even shorter wavelengths are requested by the scientific community.

One of the most promising ways to extend the photon energy range of high-gain X-ray FELs is to use harmonic lasing which is the FEL instability at an odd harmonic of the $\infty$ planar undulator [5-9] developing independently from the

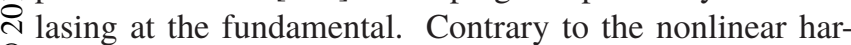
(2) monic generation $[1,6,7,10-13]$ (which is driven by the fundamental in the vicinity of saturation), harmonic lasing can provide much more intense, stable, and narrow-band radiation if the fundamental is suppressed. The most attractive feature of saturated harmonic lasing is that the spectral brightness of a harmonic is comparable to that of the fundamental [9].

Another interesting option, proposed in [9], is the possibility to improve spectral brightness of an X-ray FEL by the combined lasing on a harmonic in the first part of the undulator (with an increased undulator parameter K) and on the fundamental in the second part of the undulator. Later this concept was named Harmonic Lasing Self-Seeded FEL (HLSS FEL) [14]. Even though this scheme is not expected $\Rightarrow$ to provide an ultimate monochromatization of the FEL radiation as do self-seeding schemes using optical elements

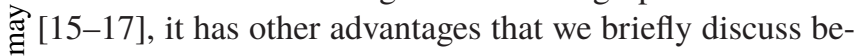
low in the paper.

Harmonic lasing was initially proposed for FEL oscillators [18] and was tested experimentally in infrared and Eisible wavelength ranges [19-22]. It was, however, never demonstrated in high-gain FELs and at a short wavelength. * evgeny.schneidmiller@ desy.de
In this paper we present the first successful demonstration of this effect at the second branch of the soft X-ray FEL user facility FLASH [23] where we managed to run HLSS FEL in the wavelength range between $4.5 \mathrm{~nm}$ and $15 \mathrm{~nm}$.

\section{HARMONIC LASING}

Harmonic lasing in single-pass high-gain FELs [5-9] is the amplification process of higher odd harmonics developing independently of each other (and of the fundamental harmonic) in the exponential gain regime. In the case of a SASE FEL the fluctuations of the beam current with frequency components in the vicinity of a wavelength

$$
\lambda_{h}=\frac{\lambda_{\mathrm{w}}\left(1+K^{2}\right)}{2 h \gamma^{2}} \quad h=1,3,5 \ldots
$$

serve as an input signal for amplification process. Here $\lambda_{\mathrm{w}}$ is the undulator period, $\gamma$ is relativistic factor, $h$ is harmonic number, and $K$ is the rms undulator parameter:

$$
K=0.934 \lambda_{\mathrm{w}}[\mathrm{cm}] B_{\mathrm{rms}}[\mathrm{T}],
$$

$B_{\text {rms }}$ being the rms undulator field (peak field divided by $\sqrt{2}$ for a planar undulator with the sinusoidal field).

An advantage of harmonic lasing over lasing on the fundamental at the same wavelength can be demonstrated for the case of a gap-tunable undulator. In this case one uses a higher K-value for harmonic lasing, i.e. for the lasing on the fundamental one has to reduce $\mathrm{K}$ to the value $K_{r e}$ :

$$
K_{r e}^{2}=\frac{1+K^{2}}{h}-1 .
$$

Obviously, $K$ must be larger than $\sqrt{h-1}$.

Then one can derive a ratio of the gain length of the fundamental, $L_{g}^{(1)}$, to the gain length of a harmonic $L_{g}^{(h)}$ [9]:

$$
\frac{L_{g}^{(1)}}{L_{g}^{(h)}}=\frac{h^{1 / 2} K A_{J J h}(K)}{K_{r e} A_{J J 1}\left(K_{r e}\right)} .
$$

Here $A_{J J h}(K)=J_{(h-1) / 2}\left(\frac{h K^{2}}{2\left(1+K^{2}\right)}\right)-J_{(h+1) / 2}\left(\frac{h K^{2}}{2\left(1+K^{2}\right)}\right)$ is the coupling factor for harmonics with $J_{n}$ being Bessel functions.

The formula (3) is obtained in the frame of the threedimensional theory including diffraction of the radiation, emittance, betatron motion (and for an optimized betafunction) but assuming a negligible energy spread. The plot of the ratio of gain lengths (3) is presented in Fig. 1. It is clearly seen that harmonic lasing has always a shorter gain length under above mentioned conditions (and the ratio is larger than that obtained in one-dimensional model [8]). The ratio shown in Fig. 2 starts to diverge rapidly for the 


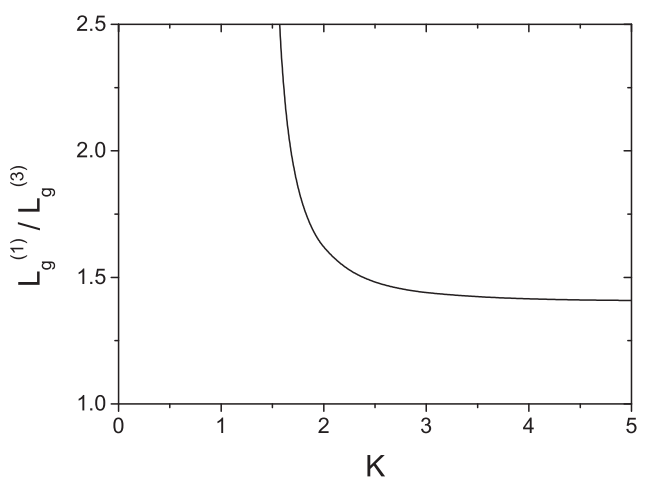

Figure 1: Ratio of the gain length of the retuned fundamental to the gain length of the third harmonic (3) for lasing at the same wavelength versus rms undulator parameter $K$. The ratio is derived in the frame of the three-dimensional theory for an optimized betafunction and negligible energy spread [9].

values of $\mathrm{K}$ approaching $\sqrt{2}$, and lasing at the fundamental becomes impossible below this point. However, there still remains a reserve in the value of parameter $\mathrm{K}$ allowing effective lasing at the third harmonic.

Amplification process of harmonics degrades with the increase of the energy spread in the electron beam more rapidly than that of the fundamental. However, in practical situations there is always the range of parameters for which the harmonic lasing still has an advantage [9].

The most attractive feature of the saturated harmonic lasing is that the spectral brightness (or brilliance) of harmonics is comparable to that of the fundamental [9]. Indeed, a good estimate for the saturation efficiency is $\lambda_{\mathrm{w}} /\left(h L_{\mathrm{sat},}, h\right)$, where $L_{\mathrm{sat},} h$ is the saturation length of a harmonic ( $h=1$ for the fundamental). At the same time, the relative rms bandwidth has the same scaling. In other words, reduction of power is compensated by the bandwidth reduction, and the spectral power remains the same. If we consider the lasing at the same wavelength on the fundamental and on a harmonic (with the retuned undulator parameter $K$ ), transverse coherence properties are about the same since they are mainly defined by the emittance-to-wavelength ratio. Thus, also the spectral brightness is about the same in both cases.

For a successful harmonic lasing to saturation, the fundamental must be suppressed. There have been different approaches proposed:

- phase shifters disrupting the fundamental but transparent for a harmonic $[8,11]$;

- spectral filtering when a filter is put into a chicane [11]; - switching between the 3rd and 5th harmonics [24,25].

Although known theoretically for a long time [5-8], harmonic lasing in high-gain FELs was never demonstrated experimentally. Moreover, it was never considered for practical applications in X-ray FELs. The situation was changed after publication of ref. [9] where it was concluded that the harmonic lasing in X-ray FELs is much more robust than usually thought, and can be effectively used in the existing

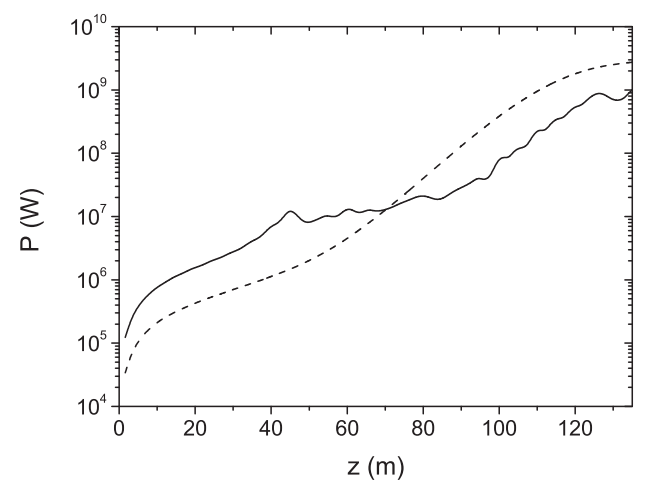

Figure 2: An example for the European XFEL. Averaged peak power for the fundamental harmonic (solid) and the third harmonic (dash) versus magnetic length of SASE1 undulator. The wavelength of the third harmonic is $0.2 \AA$ (photon energy $62 \mathrm{keV}$ ). The fundamental is disrupted with the help of phase shifters [11].

and future X-ray FELs. In particular, the European XFEL [26] can greatly outperform the specifications in terms of the highest possible photon energy: it can reach $60-100 \mathrm{keV}$ range for the third harmonic lasing, see Fig. 2. It was also shown [24] that one can keep sub-Ångström range of operation of the European XFEL after CW upgrade of the accelerator with a reduction of electron energy from $17.5 \mathrm{GeV}$ to $7 \mathrm{GeV}$. Another application of harmonic lasing is a possible upgrade of FLASH [27] with the aim to increase the photon energy up to $1 \mathrm{keV}$ with the present energy $1.25 \mathrm{GeV}$ of the accelerator. To achieve this goal, one should install a specially designed undulator optimized for the third harmonic lasing as suggested in Ref. [28].

\section{HARMONIC LASING SELF-SEEDED FEL}

A poor longitudinal coherence of SASE FELs [29-31] stimulated efforts for its improvement. Since an external seeding seems to be difficult to realize in X-ray regime, a so called self-seeding has been proposed [15-17]. There are alternative approaches for reducing bandwidth and increasing spectral brightness of X-ray FELs without using optical elements. One of them $[33,34]$ suggests to use chicanes inside the undulator system to increase slippage of the radiation and to establish long-range correlations in the radiation pulse. Another method was proposed in [9] and is based on the combined lasing on a harmonic in the first part of the undulator (with increased undulator parameter $\mathrm{K}$, see formula (2)) and on the fundamental in the second part. In this way the second part of the undulator is seeded by a narrow-band signal generated via a harmonic lasing in the first part. This concept was named HLSS FEL (Harmonic Lasing Self-Seeded FEL) [14]. Note that a very similar concept was proposed in [35] and was called a purified SASE FEL, or pSASE.

Typically, gap-tunable undulators are planned to be used in X-ray FEL facilities. If maximal undulator parameter $K$ is sufficiently large, the concept of harmonic lasing self-

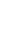

.

.

.

(n)

.
西 (2)

\section{$\frac{100}{20}$} . 商 , 敦 每 然 然

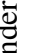
8

政




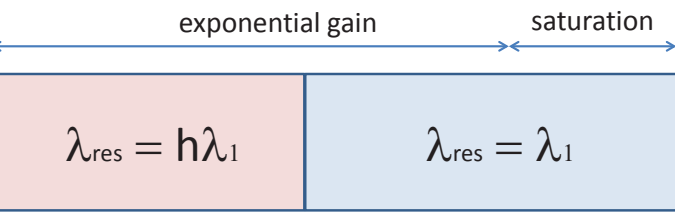

Figure 3: Conceptual scheme of a harmonic lasing self-seeded FEL.

seeded FEL can be applied in such undulators (see Fig. 3). An undulator is divided into two parts by setting two difof ferent undulator parameters such that the first part is tuned o to a $h$-th sub-harmonic of the second part which is tuned I to a wavelength of interest $\lambda_{1}$. Harmonic lasing occurs in the exponential gain regime in the first part of the undulator, also the fundamental in the first part stays well below saturation. In the second part of the undulator the fundamental is resonant to the wavelength, previously amplified as the harmonic. The amplification process proceeds in the fundamental up to saturation. In this case the bandwidth is defined by the harmonic lasing (i.e. it is reduced by a significant factor depending on harmonic number) but the saturation power is still as high as in the reference case of lasing at the fundamental in the whole undulator, i.e. the s spectral brightness increases.

The enhancement factor of the coherence length (or, bandwidth reduction factor), that one obtains in HLSS FEL in comparison with a reference case of lasing in SASE FEL mode in the whole undulator, reads [14]:

$$
R \simeq h \frac{\sqrt{L_{\mathrm{w}}^{(1)} L_{\mathrm{sat}, h}}}{L_{\mathrm{sat}, 1}}
$$

Here $h$ is harmonic number, $L_{\mathrm{sat}, 1}$ is the saturation length in the reference case of the fundamental lasing with the lower K-value, $L_{\mathrm{w}}^{(1)}$ is the length of the first part of the undula${ }^{2}$ tor, and $L_{\mathrm{sat}, h}$ is the saturation length of harmonic lasing.

We notice that it is beneficial to increase the length of the first part of the undulator. Since it must be shorter than the saturation length of the fundamental harmonic in the first s section, one can consider delaying the saturation of the funIamental with the help of phase shifters $[8,9]$ in order to increase $L_{\mathrm{w}}^{(1)}$. However, for the sake of simplicity, we did not use this option in our experiments.

Despite the bandwidth reduction factor (4) is significantly smaller than that of self-seeding schemes using optical elements [15-17], the HLSS FEL scheme is very simple and o robust, and it does not require any additional installations, i.e. it can always be used in existing or planned gap-tunable $\exists$ undulators with a sufficiently large K-value.

One more advantage of the HLSS FEL scheme over the SASE FEL (and in many cases over a self-seeded FEL) is the possibility of a more efficient use of a post-saturation taper [36-38] for an improved conversion of the electron beam power to the FEL radiation power [14,39]. Numerical simulations of the HLSS FEL were presented in [14] for the
European XFEL [26] and in [39] for FLASH [27]. In this paper we report on the operation of the harmonic lasing selfseeded FEL.

\section{OPERATION OF HLSS FEL AT FLASH}

The first soft X-ray FEL user facility FLASH $[1,27]$ was upgraded to split the electron pulse trains between the two undulator lines so that the accelerator with maximum energy of $1.25 \mathrm{GeV}$ now drives both lines. In a new separate tunnel, a second undulator line, called FLASH2, with a variable-gap undulator was installed, while a new experimental hall has space for up to six experimental stations [23]. The gap-tunable undulator of FLASH2 consists of twelve $2.5 \mathrm{~m}$ long sections with the undulator period of 3.14 $\mathrm{cm}$ and the maximum rms K-value about 1.9. This makes it possible (see formula (2)) to study the HLSS FEL scheme with the 3rd harmonic seeding.

\section{First Lasing at $7 \mathrm{~nm}$}

On May 1, 2016 we were able to successfully perform the first test of HLSS FEL at FLASH2. Electron energy was $948 \mathrm{MeV}$, charge $0.4 \mathrm{nC}$. Initially we tuned 10 undulator sections to a standard SASE, operating in the exponential gain regime at the wavelength of $7 \mathrm{~nm}$ ( $\mathrm{rms} \mathrm{K}$ parameter was 0.73 ); the pulse energy was $12 \mu \mathrm{J}$. Then we detuned the first section, tuned it to the third subharmonic (rms K was 1.9) and scanned it around $21 \mathrm{~nm}$. We repeated the measurements with the first two sections, and then with the first three sections. Note that the fundamental at $21 \mathrm{~nm}$ was also in the exponential gain regime, pulse energy after three undulator sections was $40 \mathrm{~nJ}$, i.e. it was far away from saturation (which was achieved at the $200 \mu \mathrm{J}$ level). This means, in particular, that the nonlinear harmonic generation in the first part of the undulator is excluded.

One can see from Fig. 4 that the effect is essentially resonant. For example, in the case when three undulator sections were scanned, the ratio of pulse energies at the optimal tune, $21.1 \mathrm{~nm}$, and at the tune of $20 \mathrm{~nm}$ is $51 \mu \mathrm{J} / 0.3 \mu \mathrm{J}=$ 170 . We claim that there can be only one explanation of the effect that we observe in Fig. 4: FEL gain at $7 \mathrm{~nm}$ is strongly reduced as soon as the first part of the undulator is detuned, and then the gain is recovered (and becomes even larger) due to the 3rd harmonic lasing in the first part as soon as the resonant wavelength is $21 \mathrm{~nm}$.

We should stress that the pulse energy with three retuned undulator sections $(51 \mu \mathrm{J})$ is significantly larger than that in the homogeneous undulator tuned to $7 \mathrm{~nm}$ (it was $12 \mu \mathrm{J}$ ). This is because the gain length of harmonic lasing is shorter than that of the fundamental tuned to the same wavelength (see formula (3), Fig. 2 and refs. [8, 9, 14]). A rough estimate gives us the ratio of gain lengths about 1.4 which is somewhat smaller thah the ratio in Fig. 1. The difference can be explained by a contribution of the energy spread (not included in Fig. 1) and by the fact that the undulator betafunction was larger than an optimum value. 


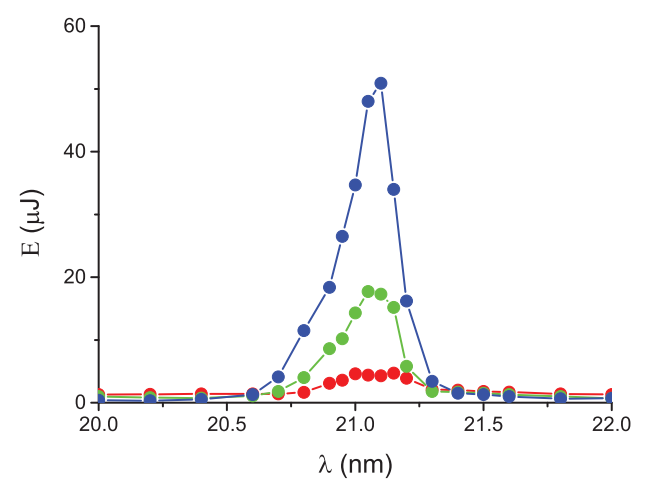

Figure 4: Scan of the resonance wavelength of the first part of the undulator consisting of one undulator section (red), two sections (green), and three sections (blue). Pulse energy is measured after the second part of the undulator tuned to $7 \mathrm{~nm}$.

\section{Improvement of the Longitudinal Coherence at $11 \mathrm{~nm}$}

We continued the studies of the HLSS FEL at FLASH2 in June 2016. Since the electron energy was different (757 $\mathrm{MeV}$ ), we lased at another wavelength, $11 \mathrm{~nm}$. We also used a different charge, $0.25 \mathrm{nC}$, in this experiment. The undulator settings were similar to the previous case: we used ten undulator modules, rms K-parameter was 0.73 in SASE mode and 1.9 in the first part of the undulator in HLSS mode. The difference with the previous measurements was that we detuned four undulator modules in HLSS regime.

In the same way as in the previous experiment, we performed the scan of the K parameter in the first part of the undulator and saw a resonance behavior again. In combination with the fact that the fundamental at $33 \mathrm{~nm}$ was by three orders of magnitude below saturation this proves that we had harmonic lasing in the first part of the undulator. The pulse energies were $11 \mu \mathrm{J}(53 \mu \mathrm{J})$ in SASE (HLSS) mode.

In Fig. 5 we present the averaged spectra for two study cases: SASE FEL with ten undulator modules and HLSS FEL with four modules tuned to $33 \mathrm{~nm}$ and six modules tuned to $11 \mathrm{~nm}$. Let us note that a per cent level discrepancy between the measured wavelength (about $10.9 \mathrm{~nm}$ ) and the wavelength expected by the undulator server $(11 \mathrm{~nm})$ comes from the fact that the server uses electron energy calculated from the RF vector sum and not from a direct measurement of the electron beam energy. However, the red shift of the radiation for the HLSS configuration with respect to the SASE case is real and can be explained by the fact that a seeded FEL radiates more efficiently in the case of a small red shift [31].

The spectra in Fig. 5 are the results of averaging over 50 single-shot spectra in each case. One can see that HLSS FEL indeed has a smaller bandwidth, $0.31 \%$, as compared to $0.41 \%$ in the case of SASE FEL. The bandwidth reduction factor is 1.3 from this measurement. The spectral power, however, differs by a factor of six due to an additional increase of pulse energy in HLSS regime. This happens be-

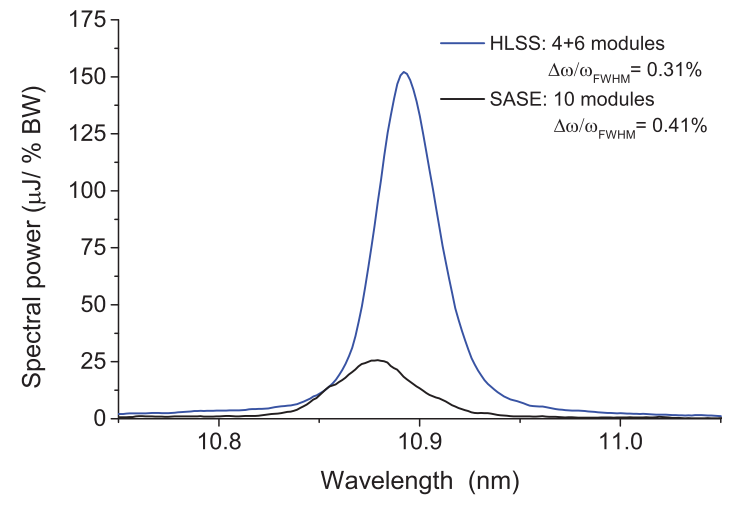

Figure 5: Spectral density of the radiation energy for HLSS FEL configuration (blue) and for SASE FEL (black).
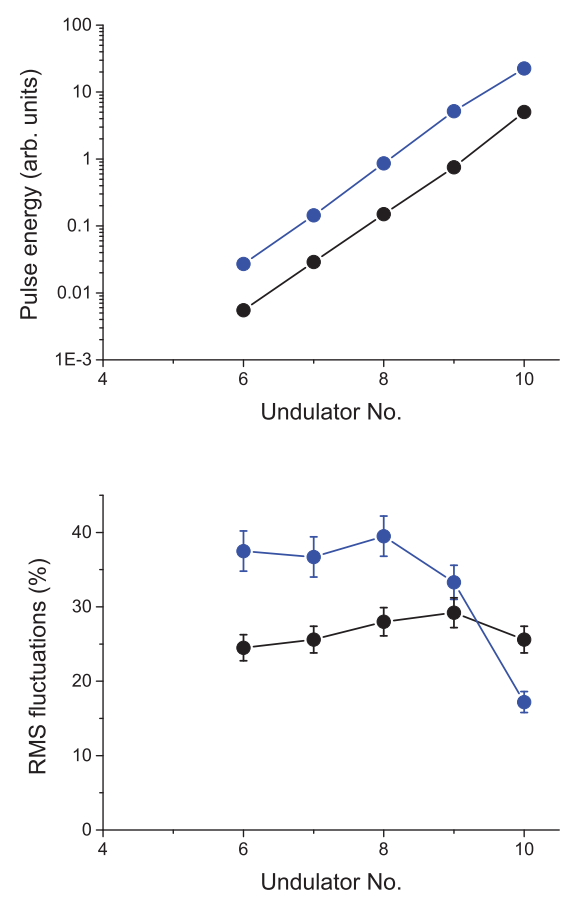

Figure 6: Radiation pulse energy (left plot) and pulse energy fluctuations (right plot) in the second part of the undulator for HLSS (blue) and for SASE (black). Small aperture in front of the MCP detector is used in this measurement.

cause 3rd harmonic lasing at $11 \mathrm{~nm}$ has a shorter gain length than lasing at the same wavelength on the fundamental.

An expected bandwidth reduction factor (or coherence enhancement factor) $R$ from formula (4) can be estimated at 1.7. The discrepancy can in a general case be explained by the energy jitter and/or energy chirp in the electron beam. The energy jitter effect is supposed to give a small contribution to the spectrum broadening since the FLASH accelerator was quite stable during the measurement, the energy stability can be estimated at the level of a few $10^{-4}$. A contribution of the energy chirp, however, being converted to a frequency chirp within an FEL pulse, can be significant.

(1)

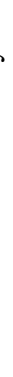


Another method of determination of an improvement of $\ddot{\Phi}$ the longitudinal coherence (independent of the presence of the frequency chirp in FEL pulses) is based on statistical measurements of the FEL pulse energy along the undulator length. It is well known $[30,31]$ that in high-gain linear regime the radiation from a SASE FEL has a statistics Ð of a completely chaotic polarized light [32]. Shot-to-shot $\leftarrow$ rms fluctuations of the FEL pulse energy $\sigma$ are connected with the number of modes by a simple relation: $M=1 / \sigma^{2}$. Number of modes can be represented as a product of the numbers of longitudinal, $M_{L}$, and transverse, $M_{T}$, modes. The latter is usually close to one, $M_{T} \simeq 1.1-1.2$ when a SASE FEL is well designed and optimized [40,41]. If one uses a small aperture to select only the central part of the FEL beam, the pulse energy fluctuations are a measure of the number of the longitudinal modes [42]: $M_{L}=1 / \sigma^{2}$. For a given FEL pulse length, the coherence length $L_{c o h}$ is inversely proportional to the number of the longitudinal modes, $M_{L}$. Making a reasonable assumption that the FEL pulse length is the same in both cases, HLSS and SASE, we end up with a simple ratio of coherence lengths for these two cases:

$$
R=\frac{L_{c o h}^{H L S S}}{L_{c o h}^{S A S E}} \simeq \frac{M_{L}^{S A S E}}{M_{L}^{H L S S}}=\frac{\sigma_{H L S S}^{2}}{\sigma_{S A S E}^{2}}
$$

In Fig. 6 we present the measurements of the FEL pulse energy and its fluctuations versus undulator length for a small aperture (significantly smaller than the FEL beam 7 size) in front of the MCP detector. In both cases, HLSS 变 and SASE, the maximum of pulse energy fluctuations is $\dot{\infty}$ achieved within the part of the undulator where the measure-

(he linear regime of the FEL operation (sections 6 to 8) the fluctuations for HLSS case are visibly larger than $\dot{r}$ in the SASE case. The validity of an assumption that pulse length in both cases is the same is justified by the fact that $\checkmark$ both FEL configurations were driven by the same electron of beam under the same conditions. We did the measurements twice for each configuration to make sure that the results E are not affected by any drifts in the accelerator. Thus, we E can conclude that in the HLSS case we had a smaller number of modes, or a larger coherence length. Using formula (5) with the fluctuations measured behind the 8th undulator $\Xi$ section for HLSS and the 9th section for SASE (at these po\& sitions with the largest fluctuations we have a similar gain $\approx$ in both modes), we obtain an estimate for the coherence enhancement factor in the end of the exponential gain regime: $R \simeq 1.8 \pm 0.3$. This is in a good agreement with already presented theoretical estimate $R \simeq 1.7$ obtained from (4).

\section{A More Efficient Post-Saturation Taper at $15 \mathrm{~nm}$}

In November 2016 we set up HLSS FEL as a configuration with four first undulators tuned to $45 \mathrm{~nm}$ and the last eight undulators tuned to $15 \mathrm{~nm}$. The electron energy was

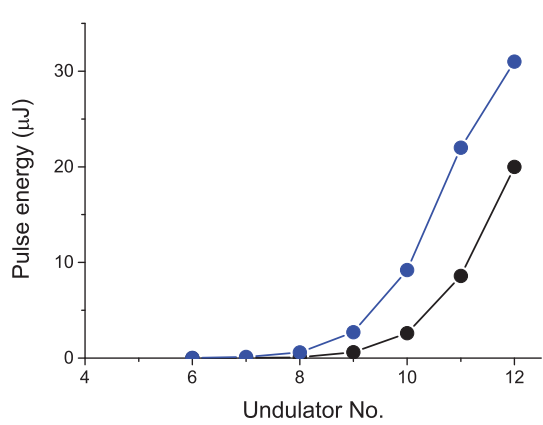

Figure 7: Radiation pulse energy versus position in the undulator for HLSS (blue) and for SASE (black). Post-saturation taper was optimized for both cases.

$645 \mathrm{MeV}$, the charge was $100 \mathrm{pC}$, the rms value of $\mathrm{K}$ was 1.9 in the first part of the undulator and 0.73 in the second part. We reached FEL saturation in SASE and HLSS modes, and applied post-saturation taper to improve FEL efficiency [36-38].

Post-saturation taper in FLASH2 undulator is implemented as a step-taper (i.e. the undulator $\mathrm{K}$ changes from section to section but is constant within a section) with linear or quadratic law. We used quadratic taper and for each mode (HLSS and SASE) optimized two parameters: beginning of tapering and the taper depth. We ended up with the following optimized parameters: beginning of tapering was in the 9th (10th) undulator and the taper depth was $0.9 \%$ $(0.7 \%)$ for HLSS (SASE). Pulse energy was enhanced for HLSS configuration from $18 \mu \mathrm{J}$ in non-tapered undulator to $31 \mu \mathrm{J}$ when post-saturation taper was applied. In case of SASE FEL the respective enhancement was from $15 \mu \mathrm{J}$ to $20 \mu \mathrm{J}$. The pulse energy versus undulator length for both operation modes is presented in Fig. 7. As it was discussed above, the improvement of post-saturation taper regime is achieved in HLSS case for two reasons: an earlier saturation and a better longitudinal coherence than in SASE case.

\section{Lasing at $4.5 \mathrm{~nm}$}

In September 2016 we were able to drive HLSS FEL by the electron beam with a higher energy, $1080 \mathrm{MeV}$, and thus obtain lasing at $4.5 \mathrm{~nm}$ in HLSS configuration. Initially, we tuned SASE regime with 12 active undulator sections (rms $\mathrm{K}$ value was 0.53 ), and could establish an onset of saturation with pulse energy at the level of $20 \mu \mathrm{J}$. Then we tuned first three sections to $13.5 \mathrm{~nm}$ (increasing rms $\mathrm{K}$ value to 1.69 ), thus providing the third harmonic signal at $4.5 \mathrm{~nm}$ for seeding the last nine undulators. The resonant behavior of the output signal together with the fact that the fundamental at $13.5 \mathrm{~nm}$ was more than three orders of magnitude below saturation proves that we had the third harmonic lasing at 4.5 $\mathrm{nm}$ in the first part of the undulator. 


\section{REFERENCES}

[1] W. Ackermann et al., Nature Photonics 1(2007)336.

[2] P. Emma et al., Nature Photonics 4 (2010) 641.

[3] T. Ishikawa et al., Nature Photonics 6 (2012) 540.

[4] A.M. Kondratenko and E.L. Saldin, Part. Accelerators 10 (1980) 207.

[5] J.B. Murphy, C. Pellegrini and R. Bonifacio, Opt. Commun. 53 (1985)197.

[6] R. Bonifacio, L. De Salvo, and P. Pierini, Nucl. Instr. Meth. A293(1990)627.

[7] Z. Huang and K. Kim, Phys. Rev. E, 62(2000)7295.

[8] B.W.J. McNeil et al., Phy. Rev. Lett. 96, 084801 (2006).

[9] E.A. Schneidmiller and M.V. Yurkov, Phys. Rev. ST-AB 15(2012)080702.

[10] H. Freund, S. Biedron and S. Milton, Nucl. Instr. Meth. A 445(2000)53.

[11] E.L. Saldin, E.A. Schneidmiller and M.V. Yurkov, Phys. Rev. $S T-A B$ 9(2006)030702.

[12] A. Tremaine et al., Phy. Rev. Lett. 88, 204801 (2002).

[13] D. Ratner et al., Phys. Rev. ST-AB 14(2011)060701.

[14] E.A. Schneidmiller and M.V. Yurkov, "Harmonic Lasing Self-Seeded FEL", in Proc. FEL2013, New York, p.700.

[15] J. Feldhaus et al., Optics. Comm. 140, 341 (1997).

[16] E.L. Saldin, Y.V. Shvyd'ko, E.A. Schneidmiller and M.V. Yurkov, Nucl. Instrum. and Methods A 475(2001)357.

[17] G. Geloni, V. Kocharyan and E.L. Saldin, Journal of Modern Optics 58(2011)1391.

[18] W.B. Colson, IEEE J. Quantum Electron. 17(1981)1417.

[19] S.V. Benson and J.M.J. Madey, Phys. Rev. A39(1989)1579.

[20] R.W. Warren et al., Nucl. Instr. Meth. A 296(1990)84.

[21] R. Hajima et al., Nucl. Instr. Meth. A 475(2001)43.

[22] N. Sei, H. Ogawa and K. Yamada, Journal of the Physical Society of Japan 79(2010)093501.

[23] B. Faatz et al., New Journal of Physics, 18(2016)062002.

[24] R. Brinkmann, E.A. Schneidmiller, J. Sekutowicz and M.V. Yurkov, Nucl. Instrum. and Methods A 768(2014)20.

[25] G. Penn, Phys. Rev. ST Accel. Beams 18, 060703 (2015).
[26] M. Altarelli et al. (Eds.), XFEL: The European X-Ray Free-Electron Laser. Technical Design Report, Preprint DESY 2006-097, DESY, Hamburg, 2006 (see also http://xfel.desy.de).

[27] S. Schreiber and B. Faatz, "The free-electron laser FLASH", High Power Laser Science and Engineering, 3, e 20 doi:10.1017/hpl.2015.16.

[28] E.A. Schneidmiller and M.V. Yurkov, Nucl. Instrum. and Methods A 717(2013)37.

[29] R. Bonifacio, C. Pellegrini and L.M. Narducci, Opt. Commun. 50(1984)373.

[30] E.L. Saldin, E.A. Schneidmiller and M.V. Yurkov, Opt. Commun. 148(1998)383.

[31] E.L. Saldin, E.A. Schneidmiller and M.V. Yurkov, "The Physics of Free Electron Lasers", Springer, Berlin, 1999.

[32] J. Goodman, Statistical Optics, (John Wiley and Sons, New York, 1985).

[33] J. Wu, J., A. Marinelli and C. Pellegrini, in Proc. 32th International Free Electron Laser Conference, Nara, Japan, 2012, p. 23, htp://w w w .jacow .org

[34] B.W.J. McNeil, N.R. Thompson and D.J. Dunning, Phys. Rev. Lett. 110(2013)134802.

[35] D. Xiang et al., Phys. Rev. ST-AB 16(2013)010703.

[36] N.M. Kroll, P.L. Morton, and M.N. Rosenbluth, IEEE J. Quantum Electron. 17, 1436 (1981).

[37] W.M. Fawley, Nucl. Instrum. Methods Phys. Res., Sect. A 375, 550 (1996)

[38] E.A. Schneidmiller and M.V. Yurkov, Phys. Rev. ST-AB 18(2015)030705.

[39] E.A. Schneidmiller and M.V. Yurkov, in Proc. IPAC16, Busan, Korea, p. 725.

[40] E.L. Saldin, E.A. Schneidmiller, and M.V. Yurkov, Opt. Commun. 281(2008)1179.

[41] E.L. Saldin, E.A. Schneidmiller, and M.V. Yurkov, New J. Phys. 12 (2010) 035010.

[42] E.A. Schneidmiller and M.V. Yurkov, in Proc. IPAC16, Busan, Korea, p. 738. 